\title{
Unravelling the Mechanisms of Electromagnetically Induced Absorption and Transparency in Open Degenerate Multilevel System: Effects of Neighboring Transitions
}

\author{
Zeeshan Ali Safdar Jadoon \\ Chosun University \\ Heung-Ryoul Noh \\ Chonnam National University \\ Jin-Tae Kim ( $\sim$ kimjt@chosun.ac.kr) \\ Chosun University
}

\section{Research Article}

Keywords: Electromagnetically Induced Absorption, Transparency, degenerate two-level system (DTLS)

Posted Date: July 15th, 2021

DOI: https://doi.org/10.21203/rs.3.rs-701938/v1

License: (c) (i) This work is licensed under a Creative Commons Attribution 4.0 International License.

Read Full License 


\title{
Unravelling the mechanisms of electromagnetically induced absorption and transparency in open degenerate multilevel system: effects of neighboring transitions
}

\author{
Zeeshan Ali Safdar Jadoon ${ }^{1}$, Heung-Ryoul Noh ${ }^{2, *}$, and Jin-Tae Kim ${ }^{1,+}$ \\ ${ }^{1}$ Department of Photonic Engineering, Chosun University, Gwangju 61452, Korea \\ ${ }^{2}$ Department of Physics, Chonnam National University, Gwangju 61186, Korea \\ *hrnoh@chonnam.ac.kr \\ +kimjt@chosun.ac.kr
}

\begin{abstract}
Optical Bloch equations with and without neighboring hyperfine states near the degenerate two-level system (DTLS) in the challenging case of ${ }^{85} \mathrm{Rb}$ D2 transition that involves the Doppler broadening effect are solved herein. The calculated spectra agree well with the experimental results obtained using the coupling-probe scheme with orthogonal linear polarizations of the coupling and probe fields. The mechanisms of electromagnetically induced absorption (electromagnetically induced transparency) for the open $F_{g}=3 \rightarrow F_{e}=2$ and 3 transitions (open $F_{g}=2 \rightarrow F_{e}=2$ and 3 transitions) are clearly determined to be the effect of the strong closed $F_{g}=3 \rightarrow F_{e}=4$ transition line (strong closed $F_{g}=2 \rightarrow F_{e}=1$ transition line) based on the comparisons of the calculated absorption profiles of a DTLS without neighboring states and those of all levels with neighboring states depending on the coupling and probe power ratios. The crucial factors established based on comparisons of the calculated absorption profiles of DTLS with and without neighboring states, which enhance or reduce coherence effects and result in transformation between electromagnetically induced absorption and electromagnetically induced transparency, are the power ratios between coupling and probe beams, openness of the excited state, and effects of the neighboring states due to the Doppler broadening in a real atomic system.
\end{abstract}

\section{Introduction}

Since the initial reports on electromagnetically induced absorption (EIA) ${ }^{1}$ and electromagnetically induced transparency $(\text { EIT })^{2,3}$, many studies ${ }^{4-19}$ have been conducted on the transitions between EITs and EIAs and the mechanisms of transitions of EITs and EIAs due to quantum coherence and interference between coupled atomic states in coupling-probe and Hanle-type experiments. However, the mechanisms of transitions between EIAs and EITs depending on powers, polarization configurations of coupling and probe beams, openness of the transitions, and neighboring effects with Doppler broadening are still unclear ${ }^{4-19}$. The mechanisms of EIA transitions from EITs depending on coupling and probe powers in the case of the $F_{e}=F_{g}-1$ open system of the ${ }^{85} \mathrm{Rb} \mathrm{D} 2$ line have not been elucidated, and such EIAs at weak coupling and probe powers have not been observed to date.

Three necessary EIA conditions for EIAs by Lezama ${ }^{20}$ for a degenerate two-level system (DTLS) were established based on first investigations of EIA resonance ${ }^{1}$ using coupling and probe lasers: (i) the ground state must be degenerate; (ii) $F_{e}=$ $F_{g}+1$; (iii) the $F_{g} \rightarrow F_{e}$ transition must be closed, without considering contributions to absorption or transmission coherence strength from neighboring states embedded in Doppler broadening profiles, power ratios between the coupling and probe beams, polarization configurations, openness of the excited state, etc.

Observations and calculations of EIAs in a DTLS that partially do not satisfy the above three conditions ${ }^{4-6,8-17,19}$ and similarly those of EITs in a DTLS that partially satisfy the above three conditions ${ }^{7,9,10,18}$ have been performed. Thus, the above conditions for EIAs are satisfied in specific scenarios such that the EIA and EIT can be observed depending on the coupling and probe powers, openness of transition, polarization configurations, and effects of neighboring states in the cases of $F_{e}=F_{g}+1, F_{e}=F_{g}$, and $F_{g}-1$.

There also exist many reports ${ }^{4-7,12,14-17}$ on the transitions between EIA and EIT depending on the general EIA conditions of the $F_{e}=F_{g}+1$ open system. However, expected EITs in the DTLS in the weak coupling and probe power regime and in the case of $F_{e} \leq F_{g}$, which violate the established EIA conditions ${ }^{20}$, transforming into genuine and symmetric EIAs considering 
the Doppler broadening effect have still not been reported. The first EIA resonances in the case of $F_{e} \leq F_{g}$ of the D1 and D2 transitions of ${ }^{85,87} \mathrm{Rb}$ were observed in the Hanle configuration, violating condition (iii) associated with closed transitions ${ }^{4}$ without any theoretical calculations for bright fluorescence profiles.

Kim et al. ${ }^{8}$ subsequently reported EIA resonances without a theoretical analysis in open systems of D1 lines of ${ }^{85} \mathrm{Rb}$ and ${ }^{87} \mathrm{Rb}$, violating conditions (ii) and (iii). These EIA results obtained only from strong coupling and probe powers above the saturation power observed by several other groups also $5,8,12,13,15-17$, result from merely enhanced absorption governed by strong coupling and probe powers, and are different from the EIAs observed and explained for the same transition configuration of $F_{e}=F_{g}-1$ and $F_{g}$ observed in this study using weak coupling and probe powers.

Chou et al. ${ }^{11}$ qualitatively interpreted these EIA anomalies in open systems using a crude model in a high-power regime without a clear quantitative explanation. Zibrov et al. ${ }^{12}$ also observed the EIA (EIT) resonances at open transitions $F_{e}=F_{g}+1$ of D1 and D2 lines of ${ }^{87} \mathrm{Rb}$ atom on artificially shifting the laser frequency toward the blue end from the center of the Doppler profile (toward the red end from the center of the Doppler profile) in both Hanle and coupling-probe experiments except in the cases of $F_{e}=F_{g}-1$ and $F_{e}=F_{g}$. Zhao et al. ${ }^{13}$ observed the EIA resonances in the strong coupling-probe regime for closed and open D2 transitions $F_{g}=4 \rightarrow F_{e}=3,4$, and 5 of $\mathrm{Cs}$ in a coupling-probe experiment with an additional laser, wherein they referred to Goren et al.'s theoretical analysis ${ }^{14}$ for the EIA anomalies at open transitions without considering the effects of neighboring states.

Auzinsh et al. observed the EIA resonances in an atomic vapor cell in the case of the open $F_{e}=F_{g}+1$ transition of ${ }^{85,87} \mathrm{Rb}$-D1 lines ${ }^{16}$. Strong coupling and probe frequencies with powers greater than $100 \mathrm{~mW} / \mathrm{cm}^{2}$ were blue-shifted to escape the neighboring dark resonance in the Hanle experiment, wherein only strong coupling and probe powers govern the absorption phenomenon. They also confirmed that the resonance fluorescence observed in the extremely thin cell that escaped neighboring effects owing to the Doppler broadening could not reveal such EIA resonances ${ }^{17}$. Auzinsh et al. also observed sign reversals of bright resonances into dark resonances at closed transitions of D2 lines of ${ }^{85} \mathrm{Rb}$ and ${ }^{87} \mathrm{Rb}$ owing to high power and temperature in the Hanle experiment ${ }^{18}$.

Grewal et al. ${ }^{19}$ reported on the influence of closed neighboring hyperfine levels with strong EIA features owing to the increased ellipticities of the polarizations of coupling and probe lasers at the $F_{e} \leq F_{g}$ open transitions of D2 lines of ${ }^{87} \mathrm{Rb}$ in the case of the Hanle configuration. However, they could not theoretically explain the absorption profiles because of the computational complexities involved when considering the Doppler effect. Performing calculations of the absorption profiles for D2 transitions of ${ }^{85} \mathrm{Rb}$ have been proven to be a particularly challenging task because of strong neighboring effects due to Doppler profiles, wherein the total absorption of D2 transition lines with the inclusion of the Doppler effect should be calculated with the inclusion of all hyperfine levels of the ground and excited states.

In this study, we deduce that clear explanations for the transition of EIT into EIA in the case of ${ }^{85} \mathrm{Rb}$ D2 transition can be obtained based on a comparison of the calculated absorption profiles of DTLS without neighboring states and all energy levels of neighboring states with respect to the power ratios between the coupling and probe powers. To investigate the power effects on coherences, the coupling-probe experiment with two orthogonal linear polarizations from a single laser combined with two acousto-optic modulators (AOMs), instead of the Hanle-type experiment, is more suitable because the power ratios of the coupling and probe lasers and a wide scanning of the frequency in the Hanle-type experiment cannot be obtained.

\section{Results}

We compare the theoretical results while considering resonant and all neighboring transitions resulting in EIAs and EITs with the experimental results. We theoretically and experimentally investigate the resonance spectra for two spectrally unresolved groups of hyperfine transitions of ${ }^{85} \mathrm{Rb} \mathrm{D} 2$ lines, i.e., $F_{g}=3 \rightarrow F_{e}=2,3$, and 4 and $F_{g}=2 \rightarrow F_{e}=1,2$, and 3, with a Ppolarized weak-probe-beam power of $15 \mu \mathrm{W}$ and S-polarized coupling beam with a scanning frequency of $5 \mathrm{MHz}$ both at a low coupling power of $50 \mu \mathrm{W}$ and strong coupling power of $4 \mathrm{~mW}$, as shown in Fig. 1 and Fig. 2. For simplicity, we use simplified notations for the transitions; e.g., the $F_{g}=3 \rightarrow F_{e}=4$ transition is expressed as $3 \rightarrow 4^{\prime}$ where unprimed (primed) integers represent the angular momentum quantum numbers in the ground (excited) states.

Figure 1 presents a comparison of the calculated spectra considering (a) a pure DTLS with transitions resonant at $3 \rightarrow 2^{\prime}$, $3 \rightarrow 3^{\prime}$, and $3 \rightarrow 4^{\prime}$; (b) a transition resonant at $3 \rightarrow 2^{\prime}$ with neighboring hyperfine transitions of $3 \rightarrow 3^{\prime}$ and $4^{\prime}$, a transition resonant at $3 \rightarrow 3^{\prime}$ with neighboring hyperfine transitions of $3 \rightarrow 2^{\prime}$ and $4^{\prime}$, and a transition resonant at $3 \rightarrow 4^{\prime}$ with neighboring hyperfine transitions of $3 \rightarrow 2^{\prime}$ and $3^{\prime}$; and (c) experimentally measured spectra with transitions resonant at $3 \rightarrow 2^{\prime}, 3 \rightarrow 3^{\prime}$, and $3 \rightarrow 4^{\prime}$ for two different weak $(50 \mu \mathrm{W})$ and strong $(4 \mathrm{~mW})$ coupling powers as shown in the top and bottom traces, respectively.

First, we explain the cases of a weak coupling power as shown in the top trace of Fig. 1. In pure two-level calculations on the left side of the top trace, a closed $3 \rightarrow 4^{\prime}$ transition satisfies all three necessary EIA conditions ${ }^{20}$ and presents a relatively strong EIA amplitude with a linewidth of $57 \mathrm{kHz}$, as expected. For open transitions $3 \rightarrow 3^{\prime}$ and $3 \rightarrow 2^{\prime}$, EIT spectra with small amplitudes and a linewidth resolution of $46 \mathrm{kHz}$ and $41 \mathrm{kHz}$ are obtained, respectively. Considering all the neighboring states 


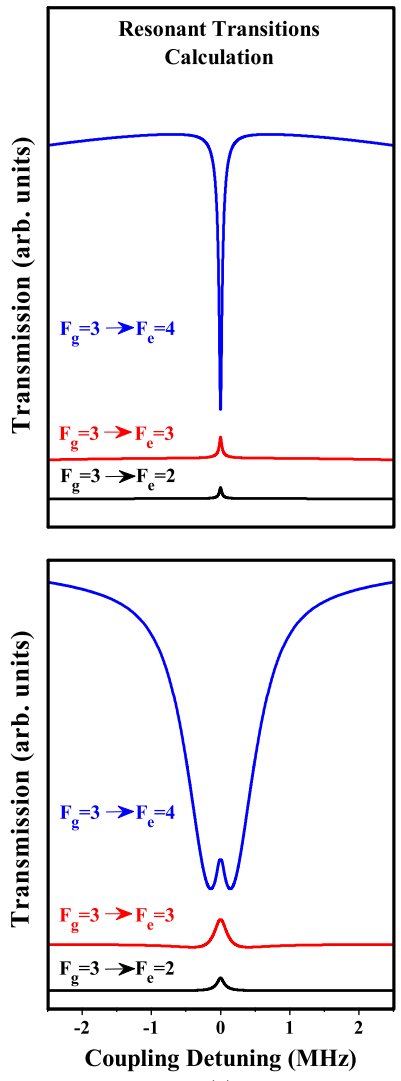

(a)

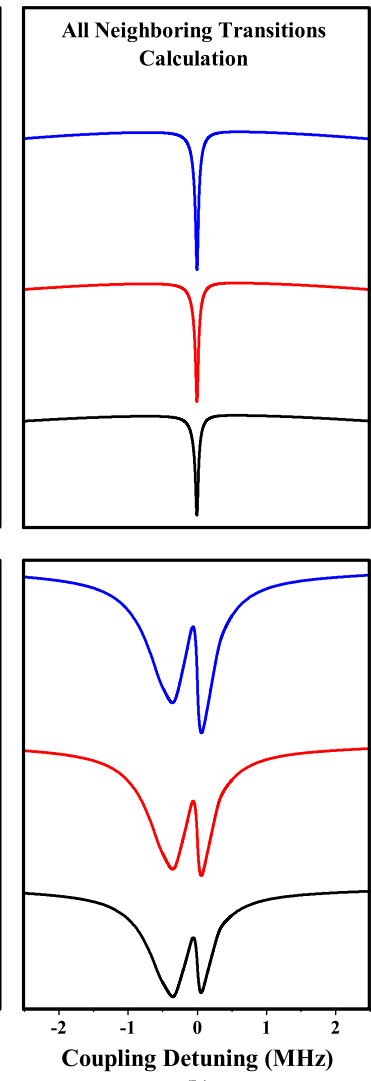

(b)
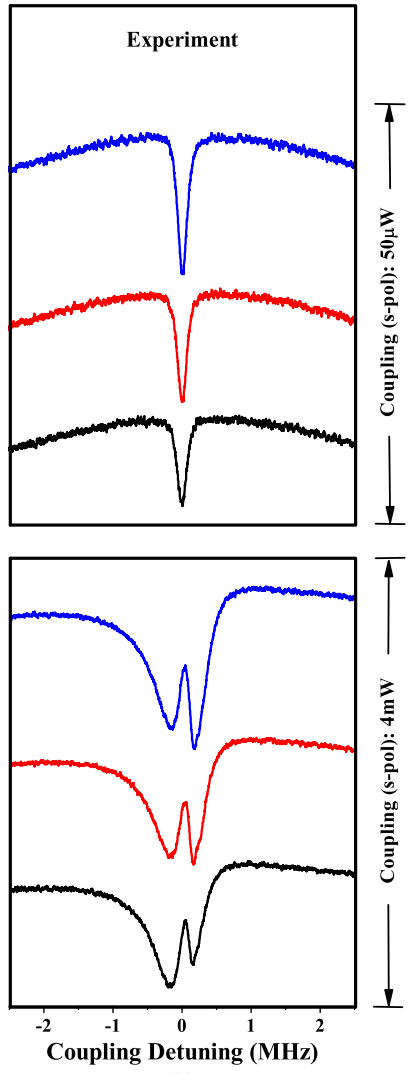

(c)

Figure 1. Comparison of calculated and measured spectra considering: (a) pure two-level resonant transitions, i.e., $3 \rightarrow 2^{\prime}$, $3 \rightarrow 3^{\prime}$ and $3 \rightarrow 4^{\prime}$, (b) a transition resonant at $3 \rightarrow 2^{\prime}$ with neighboring hyperfine transitions of $3 \rightarrow 3^{\prime}$ and $4^{\prime}$, transition resonant at $3 \rightarrow 3^{\prime}$ with neighboring transitions of $3 \rightarrow 2^{\prime}$ and $4^{\prime}$, transition resonant at $3 \rightarrow 4^{\prime}$ with neighboring transitions of $3 \rightarrow 2^{\prime}$ and $3^{\prime}$, and (c) experimentally measured spectra for resonant transitions $3 \rightarrow 2^{\prime}, 3 \rightarrow 3^{\prime}$ and $3 \rightarrow 4^{\prime}$.

near the resonant transitions of $3 \rightarrow 2^{\prime}, 3 \rightarrow 3^{\prime}$, and $3 \rightarrow 4^{\prime}$, as shown in the upper trace of Fig. 1(b), all the resonant transitions show strong EIAs with similar amplitudes owing to a nearby closed $3 \rightarrow 4^{\prime}$ transition, which exhibits strikingly similar trends in the spectra as the measured spectra, although the observed spectra are wider than the calculated ones. Measured ultra-narrow EIA dips at low coupling power are resolved with linewidths of $163 \mathrm{kHz}, 156 \mathrm{kHz}$ and $167 \mathrm{kHz}$, whereas the linewidths obtained using all the neighboring transitions calculation are $60 \mathrm{kHz}, 60 \mathrm{kHz}$, and $58 \mathrm{kHz}$ at the $3 \rightarrow 4^{\prime}, 3^{\prime}$, and $2^{\prime}$ transitions, respectively.

Second, we explain the case of a strong coupling power, as shown in the bottom trace of Fig. 1. In pure two-level calculations on the left side of the bottom trace, a closed $3 \rightarrow 4^{\prime}$ transition satisfies all three necessary EIA conditions ${ }^{20}$ and indicates a relatively strong EIA amplitude with a wide linewidth of $1.06 \mathrm{MHz}$ and splits into two broad dips at the center of the spectra, resulting in the emergence of a narrow EIT-like peak with the $117 \mathrm{kHz}$ linewidth resolution. This EIA spectra at a strong coupling power is attributed to the contributions of positive and negative atomic velocities in case of orthogonal polarizations, resulting in two identical EIA spectra separated at the right center after thermal averaging ${ }^{21}$. EIT spectra with small amplitudes and wide linewidths of $194 \mathrm{kHz}$ and $122 \mathrm{kHz}$ for open transitions $3 \rightarrow 3^{\prime}$ and $3 \rightarrow 2^{\prime}$, respectively, are obtained, as shown in lower traces of Fig. 1(a).

Including all the other adjacent transitions, such a symmetric EIA is transformed into asymmetric line profiles owing to the strong neighboring effects, which differ at the red and blue detuning regions. Considering all the neighboring states near the resonant transitions of $3 \rightarrow 2^{\prime}, 3 \rightarrow 3^{\prime}$, and $3 \rightarrow 4^{\prime}$, as shown in the lower trace of Fig. 1(b), owing to a nearby closed $3 \rightarrow 4^{\prime}$ transition, all the resonant transitions exhibit strong EIAs with similar amplitudes, exhibiting strikingly similar trends in the spectra compared to the measured spectra for the closed $3 \rightarrow 4^{\prime}$ transition. Asymmetric dips due to two separated EIAs at a strong power in the calculation (the measurement) are broad, with linewidths of $870 \mathrm{kHz}, 848 \mathrm{kHz}$, and $842 \mathrm{kHz}(607$ $\mathrm{kHz}, 606 \mathrm{kHz}$, and $603 \mathrm{kHz})$ in the red detuning and narrow with linewidths of $297 \mathrm{kHz}, 292 \mathrm{kHz}$, and $287 \mathrm{kHz}(439 \mathrm{kHz}, 402$ $\mathrm{kHz}$, and $377 \mathrm{kHz}$ ) in the blue detuning at the $3 \rightarrow 4^{\prime}, 3^{\prime}$, and $2^{\prime}$ transitions, respectively. A down-shift in relative strength of 


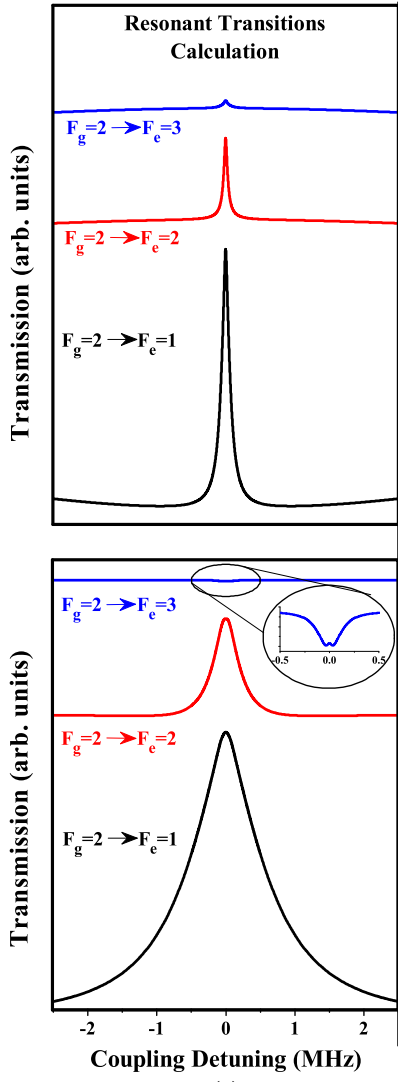

(a)

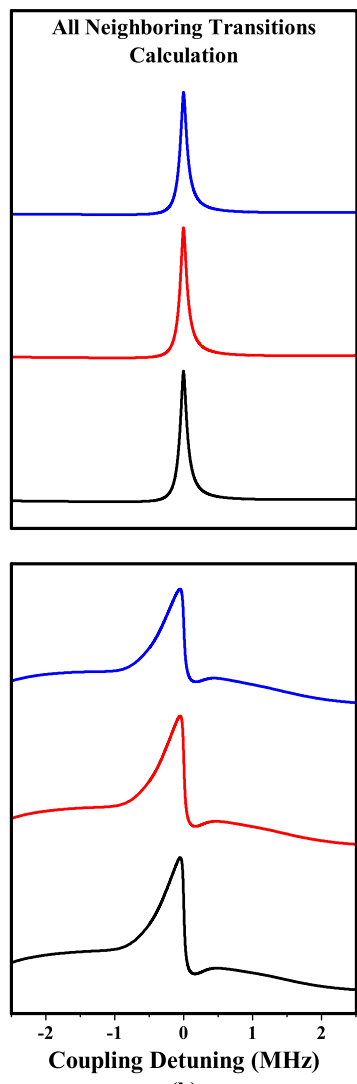

(b)

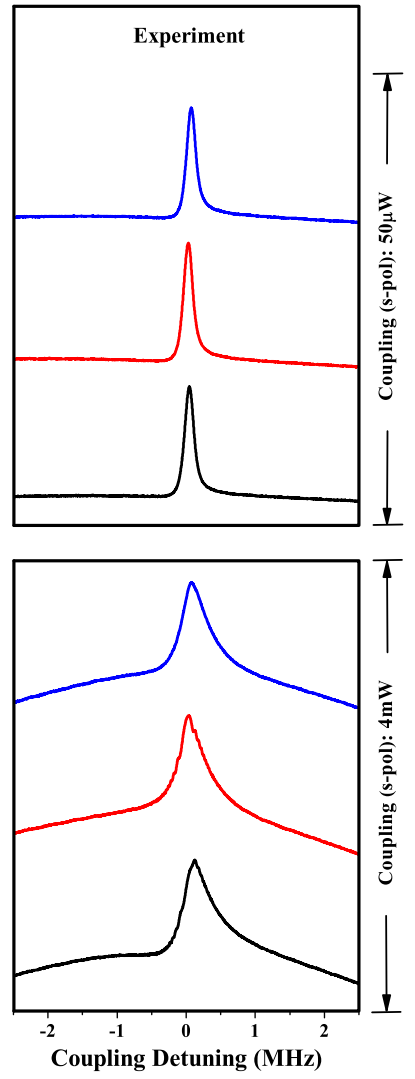

(c)

Figure 2. Comparison of calculated and measured spectra considering: (a) pure two-level resonant transitions, i.e., $2 \rightarrow 1^{\prime}$, $2 \rightarrow 2^{\prime}$ and $2 \rightarrow 3^{\prime}$, (b) transition resonant at $2 \rightarrow 1^{\prime}$ with neighboring hyperfine transitions of $2 \rightarrow 2^{\prime}$ and $3^{\prime}$, transition resonant at $2 \rightarrow 2^{\prime}$ with neighboring transitions of $2 \rightarrow 1^{\prime}$ and $3^{\prime}$, transition resonant at $2 \rightarrow 3^{\prime}$ with neighboring transitions of $2 \rightarrow 1^{\prime}$ and $3^{\prime}$, and (c) experimentally measured spectra for resonant transitions $2 \rightarrow 1^{\prime}, 2 \rightarrow 2^{\prime}$ and $2 \rightarrow 3^{\prime}$.

the amplitude is evident from the narrow blue detuned side at the $3 \rightarrow 4^{\prime}$ transition to the broad red detuned side at the $3 \rightarrow 2^{\prime}$ transition because of the frequency shifts toward those transitions.

The amplitude of the total spectra across the $3 \rightarrow 4^{\prime}, 3^{\prime}$, and $2^{\prime}$ transitions decreases with decrease in $F_{e}$. The theoretical model that considers all neighboring hyperfine states as shown in the lower trace of Fig. 1(b) and experiment as illustrated in the lower trace of Fig. 1(c) agree well in terms of contrast and asymmetry, however, there are deviations in linewidths of approximately $100 \mathrm{kHz}$ in both power regimes, which could be attributed to the effect of magnetic field, imperfect alignment of laser beams, and other effects that are not included in the calculation.

We further analyze the spectrally unresolved group of hyperfine transitions $2 \rightarrow 1^{\prime}, 2^{\prime}$, and $3^{\prime}$ of ${ }^{85} \mathrm{Rb} \mathrm{D} 2$ lines instead of $F_{g}=3$. Figure 2 presents a comparison of the calculated and measured spectra considering (a) pure two-level resonant transitions, i.e., $2 \rightarrow 1^{\prime}, 2 \rightarrow 2^{\prime}$ and $2 \rightarrow 3^{\prime}$, (b) a transition resonant at $2 \rightarrow 1^{\prime}$ with neighboring hyperfine transitions of $2 \rightarrow 2^{\prime}$ and $3^{\prime}$, a transition resonant at $2 \rightarrow 2^{\prime}$ with neighboring hyperfine transitions of $2 \rightarrow 1^{\prime}$ and $3^{\prime}$, a transition resonant at $2 \rightarrow 3^{\prime}$ with neighboring hyperfine transitions of $2 \rightarrow 1^{\prime}$ and $2^{\prime}$, and (c) experimentally measured spectra for the resonant transitions of $2 \rightarrow 1^{\prime}, 2 \rightarrow 2^{\prime}$ and $2 \rightarrow 3^{\prime}$ for both weak $(50 \mu \mathrm{W})$ and strong $(4 \mathrm{~mW})$ coupling powers as shown in the top and bottom traces, respectively.

The calculation for a DTLS in the low-power regime clearly reproduces ultra-narrow EIT resonances, as expected, at $2 \rightarrow 1^{\prime}, 2 \rightarrow 2^{\prime}$ and $2 \rightarrow 3^{\prime}$ transitions, as shown in the upper traces of Fig. 2. The obtained EIT spectra differ in relative amplitudes with the dominant EIT amplitude at the $2 \rightarrow 1^{\prime}$ transition, as shown in the upper traces of Fig. 2(a). The obtained linewidth resolutions of the EIT spectra are $90 \mathrm{kHz}, 60 \mathrm{kHz}$, and $130 \mathrm{kHz}$ at the $2 \rightarrow 3^{\prime}, 2^{\prime}$, and $1^{\prime}$ hyperfine transitions, respectively.

The EIT spectra calculated while considering all the neighboring states near the resonant state are similar in amplitude and contrast owing to the strong EIT amplitude at the $2 \rightarrow 1^{\prime}$ neighboring hyperfine transition, illustrated in the upper traces of Fig. 2(b). The trend in the measured spectra, as shown in Fig. 2(c), exhibits a striking similarity in amplitude and a contrast 
that matches well with the calculation, as shown in Fig. 2(b), while considering neighboring states. The resolved linewidth in the low coupling power regime is approximately $114 \mathrm{kHz}$ across the $2 \rightarrow 3^{\prime}, 2^{\prime}$, and $1^{\prime}$ transitions using all the neighboring calculations, as shown in Fig. 2(b); the resolved linewidths in the experiment, as presented in Fig. 2(c), are $157 \mathrm{kHz}, 158 \mathrm{kHz}$, and $163 \mathrm{kHz}$ at the $2 \rightarrow 3^{\prime}, 2^{\prime}$, and $1^{\prime}$ transitions, respectively.

In the strong coupling power regime, as shown in the lower traces of Fig. 2, the calculation that considers the resonant states shows increase in amplitudes and linewidths of the obtained spectra at the $2 \rightarrow 1^{\prime}$ and $2 \rightarrow 2^{\prime}$ hyperfine transitions. However, in violation of condition (iii) of Lezama's necessary EIA conditions, a weak-amplitude EIA with a linewidth resolution of 285 $\mathrm{kHz}$ is obtained at the $2 \rightarrow 3^{\prime}$ hyperfine transition, as highlighted in the inset image of in the lower traces of Fig. 2(a).

Asymmetric EIT spectra of relatively similar amplitudes and linewidth resolutions are obtained owing to the contributions of all the neighboring hyperfine states with the resonant transition, as shown in the lower traces of Fig. 2(b), which matches well with the trend of the measured spectra, shown in the lower traces of Fig. 2(c). However, the experimentally measured spectra at higher powers could not reflect the fine contrast, in comparison to the calculation with all the neighboring transitions shown in the lower traces of Fig. 2(b). The resolved linewidths are $388 \mathrm{kHz}, 407 \mathrm{kHz}$, and $435 \mathrm{kHz}(441 \mathrm{kHz}, 418 \mathrm{kHz}$, and $479 \mathrm{kHz}$ ) when using all the neighboring calculations (experiment).

The sign-inverting anomalies present at open transitions $3 \rightarrow 3^{\prime}$ and $3 \rightarrow 2^{\prime}$ in the previous cases, as shown in Figs. 1(b) and $1(\mathrm{c})$, are not relevant to the $2 \rightarrow 1^{\prime}, 2^{\prime}$, and $3^{\prime}$ group of spectrally resolved transitions as the EIT resonances at the $2 \rightarrow 3^{\prime}, 2^{\prime}$, and $1^{\prime}$ transitions are expected. Instead, anomalies in the relative strength of the amplitudes and linewidths at $2 \rightarrow 3^{\prime}, 2^{\prime}$, and $1^{\prime}$ are evident in the calculation on considering all the neighboring transitions and in the experiment, as shown in the lower traces of Figs. 2(b) and 2(c), respectively.

From both cases of $F_{g}=2$ and 3, we observe that the power ratios between the coupling and probe beams, openness of the excited state, and effects of neighboring states due to Doppler broadening are crucial factors in enhancing or reducing coherence effects for transformation between EIA and EIT.

\section{Discussion}

In this study, we solved the optical Bloch equations with and without neighboring hyperfine states for all hyperfine transitions of the ${ }^{85} \mathrm{Rb}$ D2 lines both in weak- and strong-coupling-power regimes while considering the Doppler effect in an ordinary vapor cell, wherein the calculated spectra matched well with the observed spectra. Theoretical models based on time-dependent density matrix equations of a degenerate (a) two-level (resonant transitions) and (b) multilevel system including all neighboring hyperfine transitions are considered for calculating coherence effects such as EIT and EIA using thermal averaging over the Doppler profile. EITs without neighboring effects in the case of open $3 \rightarrow 2^{\prime}$ and $3^{\prime}$, i.e., a DTLS that violates Lezama's EIA conditions, transforms into genuine and symmetric EIAs owing to a strong $3 \rightarrow 4^{\prime}$ EIA line with Doppler broadening in the weak coupling and probe power regime, which had remained theoretically unexplained and had not been observed previously in the case of ${ }^{85} \mathrm{Rb}$ D2 lines, while asymmetric and split EIAs are observed from calculated weak EITs in a DTLS in a weak probe and strong-coupling-power regime. The previously reported EIA resonances without clear quantitative explanations for open systems of D1 lines of ${ }^{85} \mathrm{Rb}$ and ${ }^{87} \mathrm{Rb}$ that violate conditions (ii) and (iii) of Lezama's EIA conditions are merely enhanced absorption phenomena governed by a strong coupling power ${ }^{7,8,11,13,16-18}$. These are different from the EIAs observed and explained in the same transition configuration of $F_{e}=F_{g}-1$ and $F_{g}$ observed in this work using weak coupling-probe powers.

The EITs without neighboring effects in the DTLS in the case of $2 \rightarrow 1^{\prime}\left(2 \rightarrow 2^{\prime}\right)$ remain in the EITs owing to the $2 \rightarrow 2^{\prime}$ $\left(2 \rightarrow 1^{\prime}\right)$ EIT line with the Doppler broadening in the weak probe and strong-coupling-power regime, while weak EIAs at $2 \rightarrow 3^{\prime}$ in the weak probe and strong-coupling-power regime, which violates Lezama's EIA conditions because of openness, transform into EITs. Lezama's EIA conditions in the cycling case of $3 \rightarrow 4^{\prime}$ with $F_{g}<F_{e}$ are also confirmed in strong coupling and weak probe regimes. With the comparisons of the calculated absorption profiles between a DTLS without neighboring states and all levels with neighboring states, the power ratio between probe and coupling beams, openness of the excited state, and effects of neighboring states due to Doppler broadening are crucial factors for enhancing or reducing coherence effects for transformations between EIA and EIT.

\section{Methods}

\section{Theoretical Calculation}

In this section, we describe a method of calculating the EIA spectra while considering the neighboring effect. As the details of the calculation have been reported previously ${ }^{21-25}$, we briefly present the method of calculation. The energy-level diagram of $\mathrm{D} 2$ transition line of ${ }^{85} \mathrm{Rb}$ atoms is presented in Fig. 3. The coupling and probe beams that are linearly polarized with orthogonal directions propagate in the same directions. The Rabi frequency and effective detuning of the coupling (probe) beam are denoted by $\Omega_{c}\left(\Omega_{p}\right)$ and $\delta_{c}\left(\delta_{p}\right)$, respectively. The effective detunings are given by $\delta_{c}=d_{c}-k v$ and $\delta_{p}=d_{p}-k v$, 


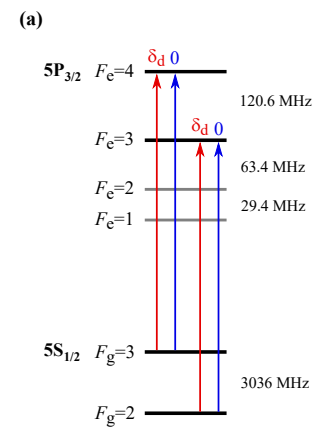

(b)

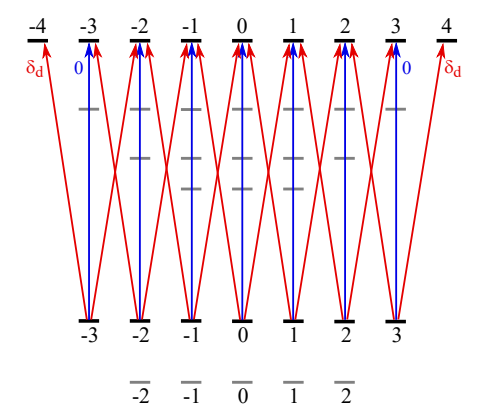

Figure 3. (a) Energy-level diagram of $\mathrm{D} 2$ transition lines of a ${ }^{85} \mathrm{Rb}$ atom, wherein the red and blue lines indicate transitions by the probe and coupling beams, respectively. (b) Transition scheme with two linearly polarized coupling and probe beams in the case of $F_{g}=3 \rightarrow F_{e}=4$ transition, where " 0 " (blue lines) and " $\delta_{d}$ " (red lines) imply the transitions excited by the coupling and probe beams, respectively. The excitations for the $F_{g}=3 \rightarrow F_{e}=2$ and 3 transitions are not shown to avoid confusion.

where $d_{c}\left(d_{p}\right)$ denotes the frequency detuning of the coupling (probe) beam, $k(=2 \pi / \lambda)$ is the wave vector, $\lambda$ is the resonance wavelength of the lasers, and $v$ is the atomic velocity. Here, $d_{c}$ and $d_{p}$ are the detunings relative to the resonance transition line under consideration.

Because the energy separation between the hyperfine states in $5 S_{1 / 2}$ ground state is approximately six times the Doppler linewidth of the atoms, we can calculate the EIA spectra for the $F_{g}=3 \rightarrow F_{e}=2,3$, and 4 transitions and $F_{g}=2 \rightarrow F_{e}=1,2$, and 3 transitions separately. In the description of the calculation method, the $F_{g}=3 \rightarrow F_{e}=2,3$, and 4 transitions are assumed. In a frame rotating with the coupling beam's frequency, the density matrix describing the internal dynamics of the atoms is given by

$$
\dot{\rho}=-\frac{i}{\hbar}\left[H_{0}+V, \rho\right]+\dot{\rho}_{\text {relax }},
$$

where $\rho$ is the density operator and $H_{0}(V)$ is the atomic (interaction) Hamiltonian.

In Eq. (1), the atomic Hamiltonian is given by

$$
\begin{aligned}
H_{0}= & -\sum_{m=-4}^{4} \hbar \delta_{c}\left|F_{e}=4, m\right\rangle\left\langle F_{e}=4, m\right| \\
& -\sum_{m=-3}^{3} \hbar\left(\delta_{c}+\Delta_{43}\right)\left|F_{e}=3, m\right\rangle\left\langle F_{e}=3, m\right| \\
& -\sum_{m=-2}^{2} \hbar\left(\delta_{c}+\Delta_{42}\right)\left|F_{e}=2, m\right\rangle\left\langle F_{e}=2, m\right|,
\end{aligned}
$$

where $\Delta_{4 j}$ is the frequency spacing between the hyperfine states $\left|F_{e}=4\right\rangle$ and $\left|F_{e}=j\right\rangle$ ( $j=2$ and 3). In Eq. (1), the interaction Hamiltonian is given by

$$
\begin{aligned}
V= & \frac{\hbar}{2} \Omega_{p} e^{-i \delta_{d} t} \sum_{q=-1}^{1} \sum_{F_{e}=2}^{4} \sum_{m=-3}^{3} a_{q} C_{3, m}^{F_{e}, m+q}\left|F_{e}, m+q\right\rangle\left\langle F_{g}, m\right| \\
& +\frac{\hbar}{2} \Omega_{c} \sum_{F_{e}=2}^{4} \sum_{m=-3}^{3} C_{3, m}^{F_{e}, m}\left|F_{e}, m\right\rangle\left\langle F_{g}, m\right|+\text { h.c. },
\end{aligned}
$$

where $F_{g}=3, a_{ \pm 1}=\mp 1 / \sqrt{2}, a_{0}=0$, and h.c. denotes Hermitian conjugate. In Eq. (3), $C_{F_{g}, m_{g}}^{F_{e}, m_{e}}$ is the normalized transition strength between states $\left|F_{e}, m_{e}\right\rangle$ and $\left|F_{g}, m_{g}\right\rangle^{22}$ and $\delta_{d}\left(\equiv \delta_{p}-\delta_{c}=d_{p}-d_{c}\right)$ is the difference in the detunings of the probe and coupling beams. In Eq. (1), $\dot{\rho}_{\text {relax }}$ represents the terms related to the relaxation mechanism such as spontaneous emission and transit time decay ${ }^{23,24}$.

As reported previously ${ }^{21-25}$, the density matrix elements are decomposed into various oscillation components. In the case of orthogonal linear polarization configuration, the coupling and probe beams excite the transitions with $\Delta m=0$ and $\Delta m= \pm 1$, respectively, where $\Delta m$ is the difference in the magnetic quantum numbers between the sublevels under consideration. In 
Fig. 3, " 0 " and " $\delta_{d}$ " imply the transitions excited by the coupling and probe beams, respectively. As stated earlier, $\delta_{d}$ is the effective frequency of the probe beam relative to the frequency of the coupling beam. The method of determining the oscillation frequencies of the density matrix elements for the orthogonal linear polarization configuration have been described in detail in Ref. 25. Herein, only an explicit expansion of the density matrix elements that are responsible for the probe absorption is given by

$$
\rho_{e_{m \pm 1}^{F_{e}}, g_{m}}=\rho_{e_{m \pm 1}^{F_{e}}, g_{m}}^{(1)} e^{-i \delta_{d} t}+\rho_{e_{m \pm 1}^{F_{e}}, g_{m}}^{(2)} e^{i \delta_{d} t},
$$

where the simplified notation for the density matrix elements is given by

$$
\rho_{e_{m_{e}}^{F_{e}}, g_{m_{g}}} \equiv\left\langle F_{e}, m_{e}|\rho| F_{g}=3, m_{g}\right\rangle \text {. }
$$

We have considered all the density matrix elements between the sublevels that are connected via photons numbers not greater than three. The absorption coefficient of the probe beam is thus given by

$$
\begin{aligned}
\alpha= & -\frac{3 \lambda^{2}}{2 \pi} \frac{N_{\mathrm{at}}}{\Omega_{p}} \int_{-\infty}^{\infty} \frac{\mathrm{d} v}{\sqrt{\pi} v_{\mathrm{mp}}} e^{-\left(v / v_{\mathrm{mp}}\right)^{2}} \\
& \times \operatorname{Im}\left[\sum_{F_{e}=2}^{4} \sum_{q=-1}^{1} \sum_{m=-3}^{3} a_{q}^{*} C_{3, m}^{F_{e}, m+q} \rho_{e_{m+q}^{F_{e}}, g_{m}}^{(1)}\right],
\end{aligned}
$$

where $N_{\text {at }}$ is the atomic number density in the cell and $v_{\mathrm{mp}}$ is the most probable speed in the cell.

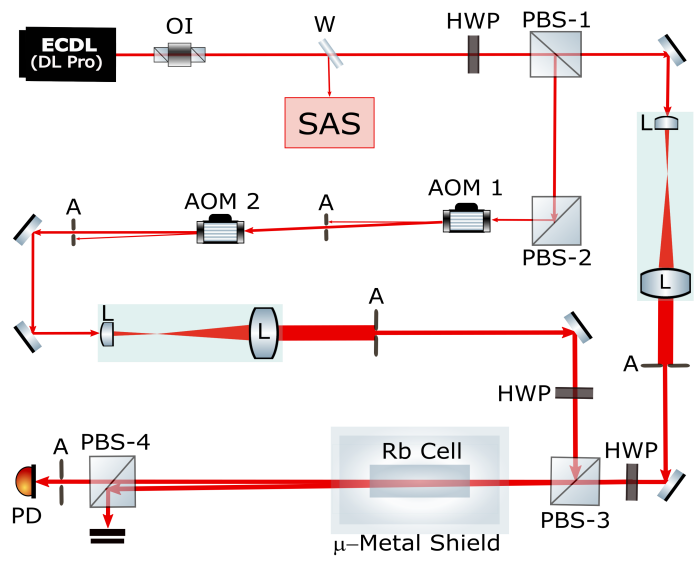

Figure 4. Experimental setup. Component symbols: OI: optical isolator; W: window; SAS: saturation absorption spectroscopy; HWP: half-wave plate; PBS: polarizing beam splitter; A: aperture; QWP: quarter-wave plate; L: lens; PD: photodiode; AOM: acousto-optic modulator.

\section{Experimental Setup}

The coupling and probe beams are generated using a single laser (DLPro, Toptica Inc.) combined with two AOMs in a single-pass configuration, as shown in Fig. 4. A combination of HWP and PBS-1 controls the ratio between the coupling and probe beam's intensities and polarizations. A P-polarized weak-probe-beam power of $15 \mu \mathrm{W}$ and an S-polarized coupling beam with a scanning frequency of $5 \mathrm{MHz}$ at a low coupling power of $50 \mu \mathrm{W}$ and strong coupling power of $4 \mathrm{~mW}$ were used. The coupling and probe beams were expanded by five times to obtain a uniform intensity across a 4-mm diameter. The coupling beam with the scanning detuning $(\Delta)$ from AOM-2 is combined with the weak probe at PBS-3 and co-propagates with orthogonal linear polarizations while maintaining an angle of intersection of approximately $0.1 \mathrm{mrad}$ through the vapor cell shielded with five layers of $\mu$-metal sheets. PBS-4 eliminates the coupling beam before the detection of the probe intensity at PD. The desired $5 S_{1 / 2} \rightarrow 5 P_{3 / 2}$ transition of ${ }^{85} \mathrm{Rb}$ D2 hyperfine line is locked using a saturation absorption spectroscopy (SAS) setup. The experiments are performed with ${ }^{85} \mathrm{Rb}$ atoms in a vapor cell at room temperature.

\section{References}

1. Akulshin, A. M., Barreiro, S. \& Lezama, A. Electromagnetically induced absorption and transparency due to resonant two-field excitation of quasidegenerate levels in rb vapor. Phys. Rev. A 57, 2996-3002, DOI: 10.1103/PhysRevA.57.2996 (1998). 
2. Harris, S. E., Field, J. E. \& Imamoğlu, A. Nonlinear optical processes using electromagnetically induced transparency. Phys. Rev. Lett. 64, 1107-1110, DOI: 10.1103/PhysRevLett.64.1107 (1990).

3. Boller, K.-J., Imamoğlu, A. \& Harris, S. E. Observation of electromagnetically induced transparency. Phys. Rev. Lett. 66, 2593-2596, DOI: 10.1103/PhysRevLett.66.2593 (1991).

4. Dancheva, Y., Alzetta, G., Cartaleva, S., Taslakov, M. \& Andreeva, C. Coherent effects on the zeeman sublevels of hyperfine states in optical pumping of Rb by monomode diode laser. Opt. Commun. 178, 103-110, DOI: https://doi.org/ 10.1016/S0030-4018(00)00643-X (2000).

5. Alzetta, G. et al. Coherent effects on the zeeman sublevels of hyperfine states at the $\mathrm{D}_{1}$ and $\mathrm{D}_{2}$ lines of Rb. J. Opt. B: Quantum Semiclassical Opt. 3, 181-188, DOI: 10.1088/1464-4266/3/3/317 (2001).

6. Failache, H., Valente, P., Ban, G., Lorent, V. \& Lezama, A. Inhibition of electromagnetically induced absorption due to excited-state decoherence in Rb vapor. Phys. Rev. A 67, 043810, DOI: 10.1103/PhysRevA.67.043810 (2003).

7. Papoyan, A., Auzinsh, M. \& Bergmann, K. Nonlinear hanle effect in Cs vapor under strong laser excitation. The Eur. Phys. J. D - At. Mol. Opt. Plasma Phys. 21, 63-71, DOI: 10.1140/epjd/e2002-00178-y (2002).

8. Kim, S. K., Moon, H. S., Kim, K. \& Kim, J. B. Observation of electromagnetically induced absorption in open systems regardless of angular momentum. Phys. Rev. A 68, 063813, DOI: 10.1103/PhysRevA.68.063813 (2003).

9. Brazhnikov, D. V. et al. Effect of the polarization of counterpropagating light waves on nonlinear resonances of the electromagnetically induced transparency and absorption in the hanle configuration. JETP Lett. 91, 625-629, DOI: 10. 1134/S0021364010120039 (2010).

10. Saprykin, E. G., Chernenko, A. A. \& Shalagin, A. M. Polarization phenomena in the transparency and adsorption effects induced by the field of counterpropagating waves. J. Exp. Theor. Phys. 119, 196-205, DOI: 10.1134/S1063776114070140 (2014).

11. Chou, H.-S. \& Evers, J. Dressed-atom multiphoton analysis of anomalous electromagnetically induced absorption. Phys. Rev. Lett. 104, 213602, DOI: 10.1103/PhysRevLett.104.213602 (2010).

12. Zibrov, A. S. \& Matsko, A. B. Induced absorption resonance on the open $F_{\mathrm{g}}=1 \rightarrow F_{\mathrm{e}}=2$ transition of the $D_{1}$ line of the ${ }^{87} \mathrm{Rb}$ atom. J. Exp. Theor. Phys. Lett. 82, 472-476, DOI: 10.1134/1.2150864 (2005).

13. Jian-Ming, Z., Yan-Ting, Z., Tao, H., Lian-Tuan, X. \& Suo-Tang, J. Observation of EIA in closed and open caesium atomic system. Chin. Phys. 14, 725-728, DOI: 10.1088/1009-1963/14/4/015 (2005).

14. Goren, C., Wilson-Gordon, A. D., Rosenbluh, M. \& Friedmann, H. Electromagnetically induced absorption due to transfer of coherence and to transfer of population. Phys. Rev. A 67, 033807, DOI: 10.1103/PhysRevA.67.033807 (2003).

15. Auzinsh, M., Ferber, R., Gahbauer, F., Jarmola, A. \& Kalvans, L. $f$-resolved magneto-optical resonances in the $D_{1}$ excitation of cesium: Experiment and theory. Phys. Rev. A 78, 013417, DOI: 10.1103/PhysRevA.78.013417 (2008).

16. Auzinsh, M., Ferber, R., Gahbauer, F., Jarmola, A. \& Kalvans, L. Nonlinear magneto-optical resonances at $D_{1}$ excitation of ${ }^{85} \mathrm{Rb}$ and ${ }^{87} \mathrm{Rb}$ for partially resolved hyperfine $f$ levels. Phys. Rev. A 79, 053404, DOI: 10.1103/PhysRevA.79.053404 (2009).

17. Auzinsh, M. et al. Nonlinear magneto-optical resonances at $D_{1}$ excitation of ${ }^{85} \mathrm{Rb}$ and ${ }^{87} \mathrm{Rb}$ in an extremely thin cell. Phys. Rev. A 81, 033408, DOI: 10.1103/PhysRevA.81.033408 (2010).

18. Auzinsh, M. et al. Conversion of bright magneto-optical resonances into dark resonances at fixed laser frequency for $D_{2}$ excitation of atomic rubidium. Phys. Rev. A 85, 033418, DOI: 10.1103/PhysRevA.85.033418 (2012).

19. Grewal, R. S. \& Pattabiraman, M. Hanle electromagnetically induced absorption in open $F_{\mathrm{g}} \rightarrow F_{\mathrm{e}} \leqslant F_{\mathrm{g}}$ transitions of the ${ }^{87} \mathrm{Rb} D_{2}$ line. J. Phys. B: At. Mol. Opt. Phys. 48, 085501, DOI: 10.1088/0953-4075/48/8/085501 (2015).

20. Lezama, A., Barreiro, S. \& Akulshin, A. M. Electromagnetically induced absorption. Phys. Rev. A 59, 4732-4735, DOI: 10.1103/PhysRevA.59.4732 (1999).

21. Rehman, H.-U., Adnan, M., Noh, H.-R. \& Kim, J.-T. Spectral features of electromagnetically induced absorption in ${ }^{85} \mathrm{Rb}$ atoms. J. Phys. B: At. Mol. Opt. Phys. 48, 115502, DOI: 10.1088/0953-4075/48/11/115502 (2015).

22. Choi, G.-W. \& Noh, H.-R. Sub-doppler DAVLL spectra of the d1 line of rubidium: a theoretical and experimental study. J. Phys. B: At. Mol. Opt. Phys. 48, 115008, DOI: 10.1088/0953-4075/48/11/115008 (2015).

23. Rehman, H. U., Mohsin, M. Q., Noh, H.-R. \& Kim, J.-T. Electromagnetically induced absorption due to transfer of coherence and coherence population oscillation for the $F_{\mathrm{g}}=3 \rightarrow F_{\mathrm{e}}=4$ transition in ${ }^{85} \mathrm{Rb}$ atoms. Opt. Commun. 381, 127-134, DOI: https://doi.org/10.1016/j.optcom.2016.06.063 (2016). 
24. Choi, G.-W. \& Noh, H.-R. Line shapes in sub-doppler davll in the ${ }^{87} \mathrm{Rb}-\mathrm{D} 2$ line. Opt. Commun. 367, 312-315, DOI: https://doi.org/10.1016/j.optcom.2016.01.073 (2016).

25. Jadoon, Z. A. S., Noh, H.-R. \& Kim, J.-T. Multiphoton nonlinear frequency mixing effects on the coherent electromagnetically induced absorption spectra of ${ }^{85} \mathrm{Rb}$ atoms under a longitudinal magnetic field: Theory and experiment. Phys. Rev. A 102, 063714, DOI: 10.1103/PhysRevA.102.063714 (2020).

\section{Acknowledgements}

This work was supported by the National Research Foundation of Korea (NRF) grant funded by the Korea government (MSIT) (No. 2020R1A2C1005499 and No. 2021R1F1A1052487).

\section{Author contributions statement}

J.-T. conceived and supervised the experiments, Z.A.S. conducted the experiments and analysed the results, H.-R. performed theoretical calculations. All authors reviewed the manuscript.

\section{Additional information}

\section{Competing interests}

The authors declare no competing interests. 